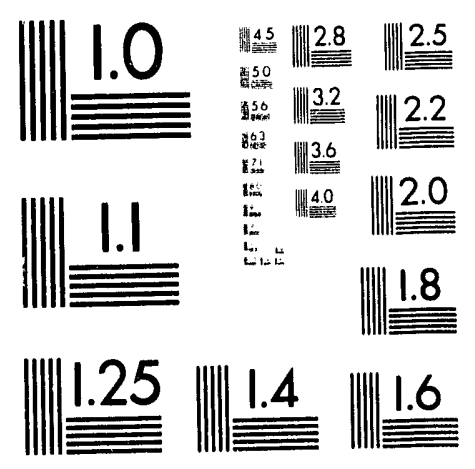



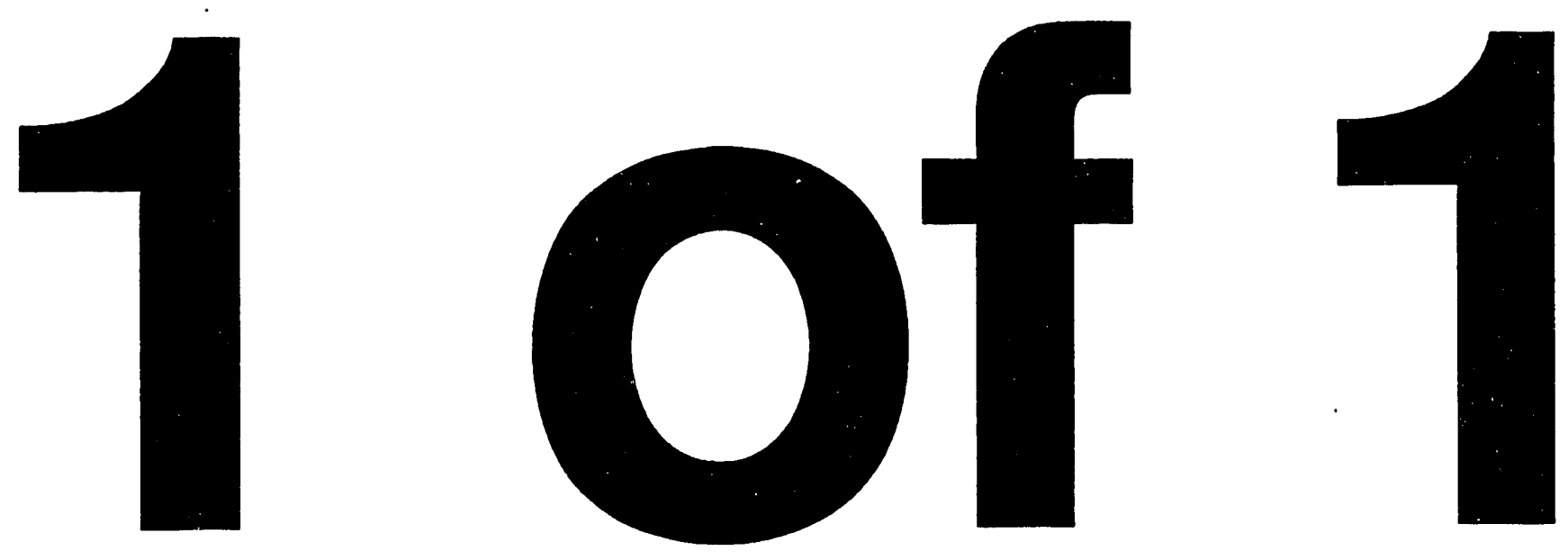
A Comparison of Film and Phosphor Scanners

Trae Chancellor

Roger A. Morris 


\title{
A COMPARISON OF FILM AND PHOSPHOR SCANNERS
}

\author{
by
}

\author{
Trae Chancellor and Roger A. Morris
}

\begin{abstract}
Signal-to-noise ratios (SNRs) and spatial distortions have been measured for three types of scanners: the Molecular Dynamics (MD) and DuPont film scanners and the MD phosphor scanner. The MD film scanner is a deployable and compact scanner that gives a peak SNR of 110 for low $(<2.0)$ optical densities (ODs), but the spatial distortions across the digitized film plane are significant. We compare this with the DuPont film scanner, which has equally good SNRs at low ODs, but very low spatial distortions. The DuPont also allows the user to define an OD range and contains a pre-scan function to find the suitable range if the user cannot input such a value; its scan times are quick, and the hardware allows for internal data averaging before being stored to disk. The MD phosphor imager has excellent low-dose capability, producing usable images at a $10-\mu \mathrm{rad}$ dose (from a 150-pkeV source) but its SNRs are low compared to the film scanner, but they can be increased by adjusting the photomultiplier tube voltage and laser radius across the scan arc.
\end{abstract}

\section{INTRODUCTION}

The Advanced Nuclear Technology Group at Los Alamos National Laboratory currentiy has two film scanners and one storage phosphor scanner that support the radiographic operations of the group: Molecular Dynamics (MD) and DuPont film scanners and an MD phosphor scanner. The DuPont NDT-70 film scanner (see Fig. 1), purchased as a high-performance research scanner, was used in earlier research. Later a more compact, rugged, and deployable film scanner, the MD 300E, was purchased for field use. While some of its scanning characteristics are not as good as the DuPont, the 300E still produces an image that can be used successfully in the field.

To further enhance field capabilities, an MD 400E phosphor scanner was purchased. This imager scans plates that are made of phosphor compounds, using a laser to excite electrons that have moved to metastable empty sites in the compound from a previous irradiation. When the electrons are freed by the laser excitation energy, they combine with one of the elements from the compound and emit light that is then collected. The amount of light that is emitted from a particular point on the screen is proportional to the amount of $\mathrm{x}$ - or gamma-ray dose received at that point. The collected light is amplified by a photomultiplier tube (PMT), digitized with an analogto-digital converter (ADC), and written to disk. Figure 2 shows both the MD 300E (right) and the $400 \mathrm{E}$ (left) side-by-side. Note that both scanners are similar in size and construction. 
These scanners all have different characteristics (such as pixel size, scanning time, SNR, and spatial distortion) that must be determined if accurate, qualitative image analysis procedures are applied to the $\mathrm{x}$-ray image. This report contains the characteristic data found for each of the three scanners.

\section{BACKGROUND}

Radiography can be used as a diagnostic tool for unknown objects. The ionizing radiation emitted from a source is attenuated by the object's mass before it deposits some of its remaining energy on a film or phosphor (fluor) plate (see Fig. 3). In the case of film, the image is stored as silver grains with the amount of silver proportional to the absorbed dose. (Additional information on film/phosphor comparisons may be found in Ref. 1.)

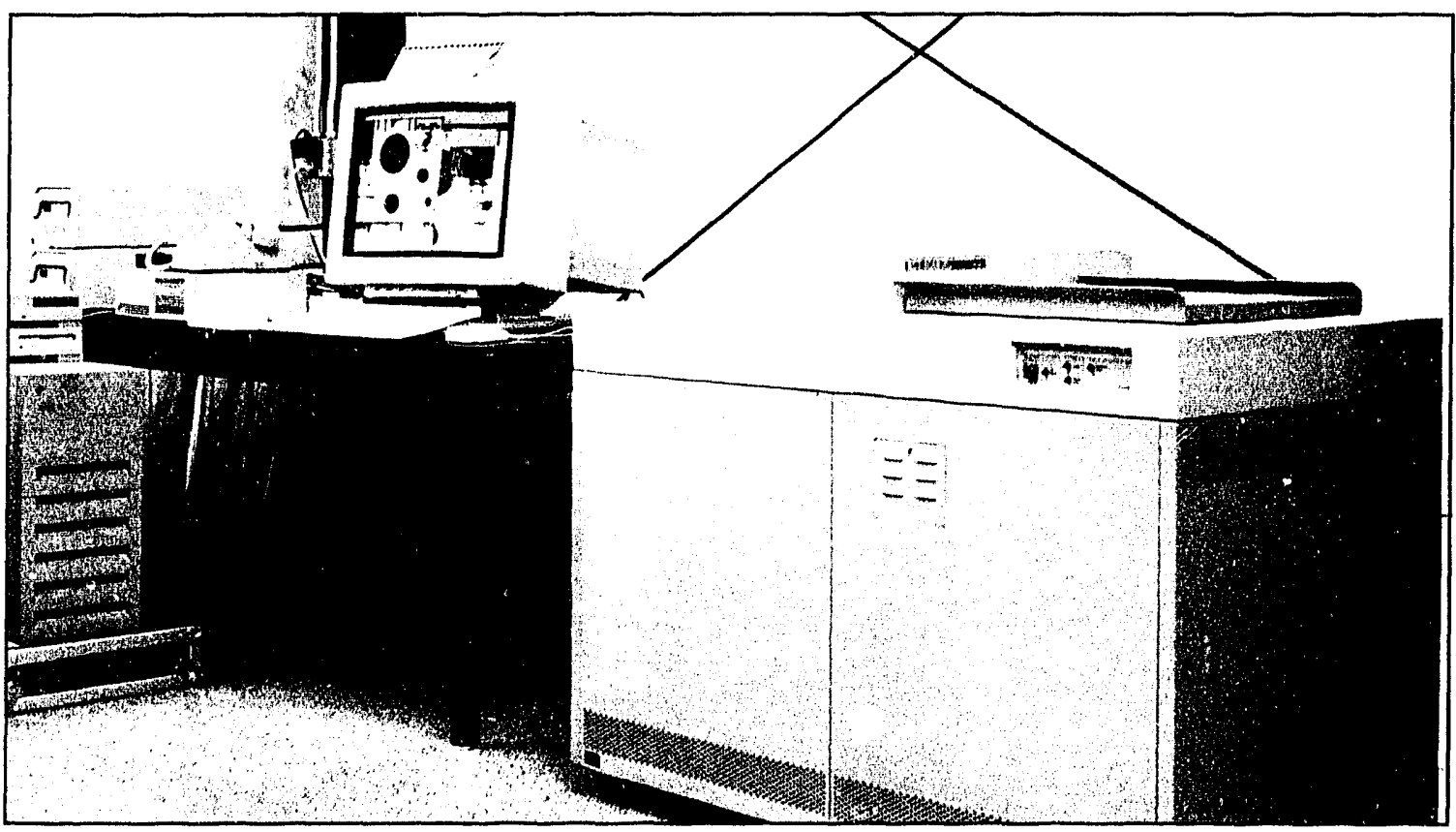

Fig. 1. DuPont NDT-70 scanner shown with Sparc workstation.

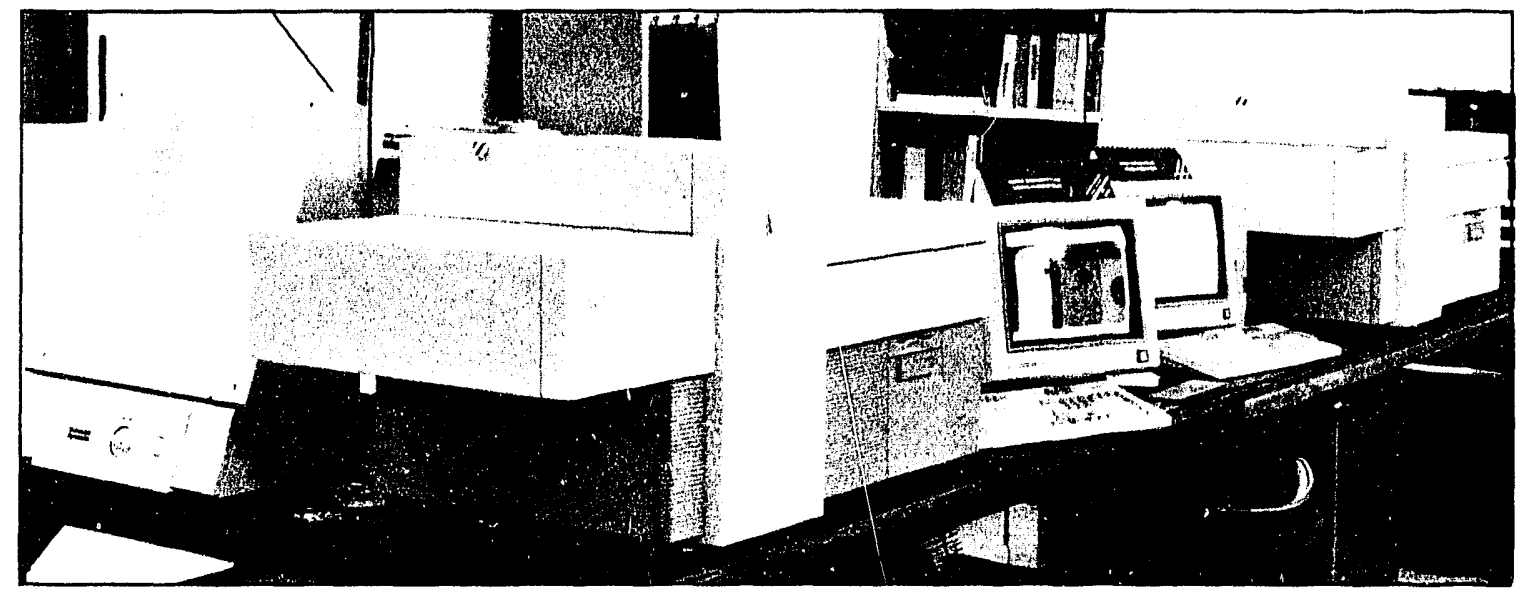

Fig. 2. Photograph of the MD 30OE (right side) and 400E (left) side-by-side. 
Both I )uPont and MI) film scanners digitize the ir images by passing light through the film and measuring the light transmission. The output from the Ml) scanner is converted to optical density (O) ) $\times 1()(k)$ before being written to disk, but the DuPont scanner outputs transmitted intensity values directly. The relationship between (OD) and transmission at at point is

$$
\mathrm{OD}=\log 10(\mathrm{~S} / \mathrm{SO})
$$

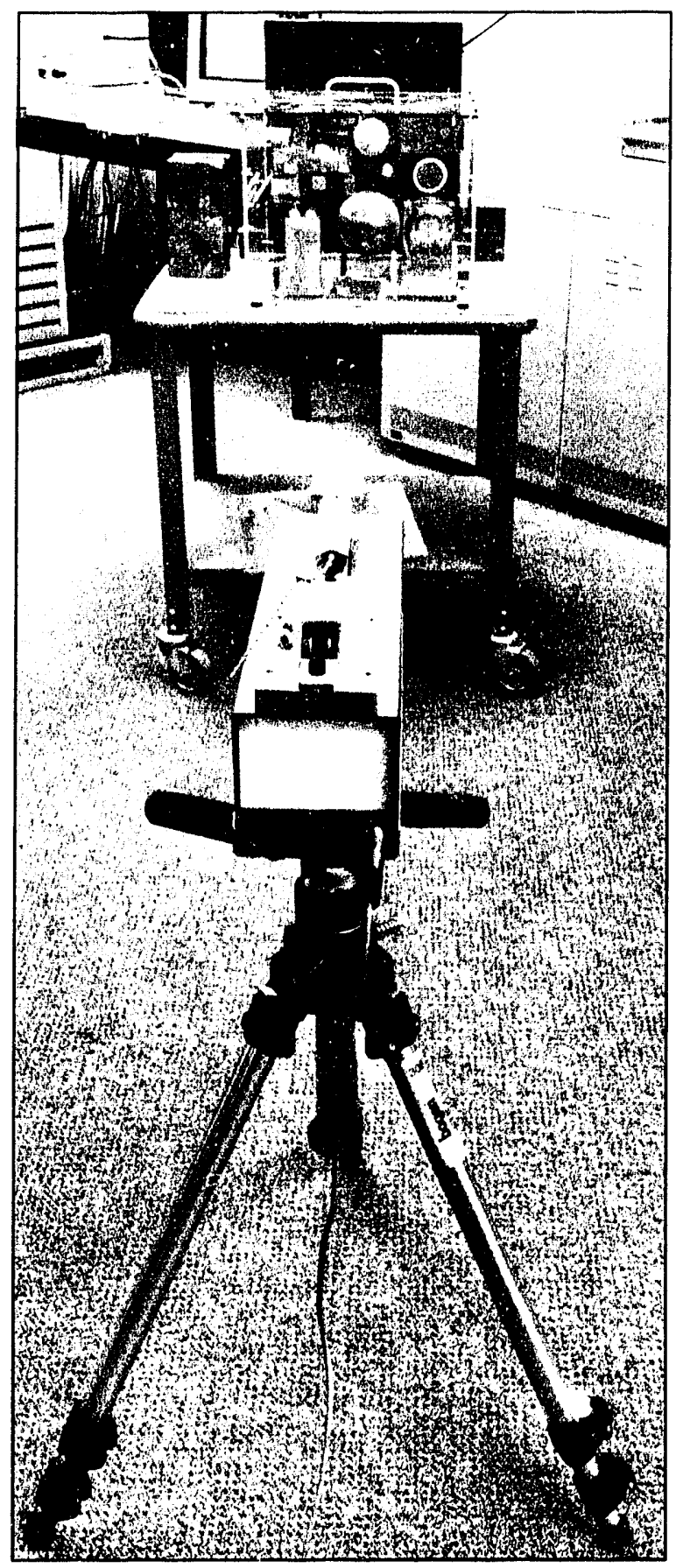

Fig. i. Setup of a radiographice experiment showing the 1.5(1)-pkV x-ray sourece (foregeroumd) aimed at an unkinown objecet (boxed) with a fluor/film cassette (barkigroumd). where So is the incident intensity value, and $S$ is the average value of transmitted intensity at the point.

The storage phosphor scanner operates differently from film scanners. Phosphors are composed of BaFBr:Eu+2 compounds. When a phosphor is exposed to radiation, the Eu+2 electrons are excited, become free, and move within the phosphor compound to emply sites. The Eu+2 compound is then oxidized and becomes Eu+3 and remains in that state until light or, in this case, a helium-neon laser (with a wavelength $\sim 633 \mathrm{~nm}$ and a spot size of $88 \mu \mathrm{m}$ ) excites the trapped electrons. ${ }^{2}$ This allows the electrons free movement once again, and they combine with Eu+3 to form Eu+2. When this occurs, blue light is emitted and collected, using an optical bundle. The stage of the phosphor scanner moves in increments of $88 \mu \mathrm{m}$, which is the diameter of the laser (it also has an option to move incrementally at $176 \mu \mathrm{m}$ ). The collected light is first converted to current with a PMT; then switched to voltage by a current-to-voltage converter; amplified; digitized, using 16 bits; and finally, the square root of the data is taken and written to disk.

To research and compare the scanners, several parameters need to be determined. The SNR can be determined for all three scanners by irradiating film and phosphor at equal doses, then digitizing them. The SNR is given by

$$
\mathrm{SNR}=\mathrm{M} / \mathrm{I},
$$

where $M$ is the mean value of the signal for at region of interest (ROI), and $\mathrm{D}$ is the standard deviation of the signal in the same ROI. To compare results, the ROI must be kept the same size for both the film and phosphor; the fluor must be uniformly 
irradiated. Further irradiations of the film/phosphor can determine the ratio as a function of dose received.

Spatial distortion, which indicates each scanner's accuracy in measuring distances from the digitized image, can be gauged by scanning a line grid template. Distances in the digitized image can be measured with an image-processing package, then compared to actual values. For film scanners, the film template can simply be scanned; however, the phosphor must first be irradiated, then have the template taped to it before being scanned. Using the same template for all scanners guarantees that the results are comparable.

\section{PROCEDURES}

\section{A. Determining Spatial Distortions}

To determine spatial distortions on all scanners, a computer-aided-design package was used to draw a mesh of $1-\mathrm{cm}^{2}$ squares on Mylar film with a precision plotter. The DuPont and MD film scanners were calibrated to establish operating parameters. The DuPont scanner was set up to scan at $70-\mu \mathrm{m}$ pixels and an OD range of $0.0-3.8$; the MD film scanner used $88-\mu \mathrm{m}$ pixels and the only OD range available. For the phosphor scanner, a phosphor plate was irradiated with $100 \mu \mathrm{rad}$ from a 150-pkeV x-ray source. The Mylar film was then taped to the front of the plate and scanned with $88-\mu \mathrm{m}$ pixels and a PMT voltage of $800 \mathrm{~V}$. The PMT voltage, which can only be changed on the phosphor scanner and not the others, was kept constant at $800 \mathrm{~V}$ for all scans.

The measured positions of the grid lines in the scanned images were compared to the actual positions on the Mylar film. The differences (errors) were plotted as a function of position within the image. The plots showed the severity of the spatial distortions along both axes for each of the scanners.

A series of signal-to-noise measurements was made over a wide dynamic range. Both the film and phosphor were irradiated by a pulsed 150-pkeV x-ray source, placed $3 \mathrm{~m}$ away, in a series of steps that ranged in value from background to 256 pulses. Each irradiation step on the film/ phosphor was a factor of 2 greater than the previous step, and the average dose from the $x$-ray source was $30 \mu \mathrm{rad} / \mathrm{pulse}$ at the film/phosphor plane. ${ }^{3}$

NDT -57 film, enclosed by NDT-9 phosphors, was chosen as the film/fluor combination. The film was developed and digitized on both MD and DuPont scanners, and the data files were analyzed on a Sparc 2 workstation, running Sunvision 1.2 software. The MD used $88-\mu \mathrm{m}$ pixels and the DuPont used 140-.'. $\mathrm{m}$ pixels. To compare the two sets of data (DuPont and MD), the DuPont values had to be converted from transmission values to $O D$, and the MD values had to be divided by 1000 (because it is multiplied by 1000 before being stored). A 220 - by 40 -pixel region of interest (ROI) was used to find the average signal and standard deviation. This was done for all irradiation steps.

The phosphor screen with the same irradiation steps as the film was scanned using $800 \mathrm{~V}$ on the PMT and $88-\mu \mathrm{m}$ pixels. The image was then transferred to the Sparc 2 , where data was squared and divided by 21025 to restore original data. The same 220 - by 40 -pixel ROI was used to calculate three SNR per step, and the data was averaged and plotted against the number of pulses. 


\section{EXPERIMENTAL RESULTS}

\section{A. Spatial Distortion}

Overall spatial distortions within the film/fluor plane were calculated by comparing the nominal value of pixels per centimeter with the measured values from the digitized image. The MD film scanner showed large spatial distortions across the short axis (Fig. 4a). The differences between actual and measured values were consistently high, averaging 118.1 pixels $/ \mathrm{cm}$ with a 2.20-pixels $/ \mathrm{cm}$ std dev. For the long axis, the measured number of pixels per centimeter averaged 113.6, with a 1.73 std dev. Although measured and actual pixels per centimeter for the long axis were close, there were some significant distortions (Fig. 4b).

The MD phosphor scanner had good agreement between measured and actual values, evidencing low spatial distortions within the film plane. The short axis averaged 113.4 pixels $/ \mathrm{cm}$ with a $1.5 \mathrm{std} \mathrm{dev} \mathrm{(see} \mathrm{Fig.} \mathrm{5a);} \mathrm{the} \mathrm{long} \mathrm{axis} \mathrm{averaged} 113.4$ pixels/cm with a $1.12 \mathrm{std} \mathrm{dev}$ (Fig. 5b). No large reproducible distortions were found for this scanner.

(a)

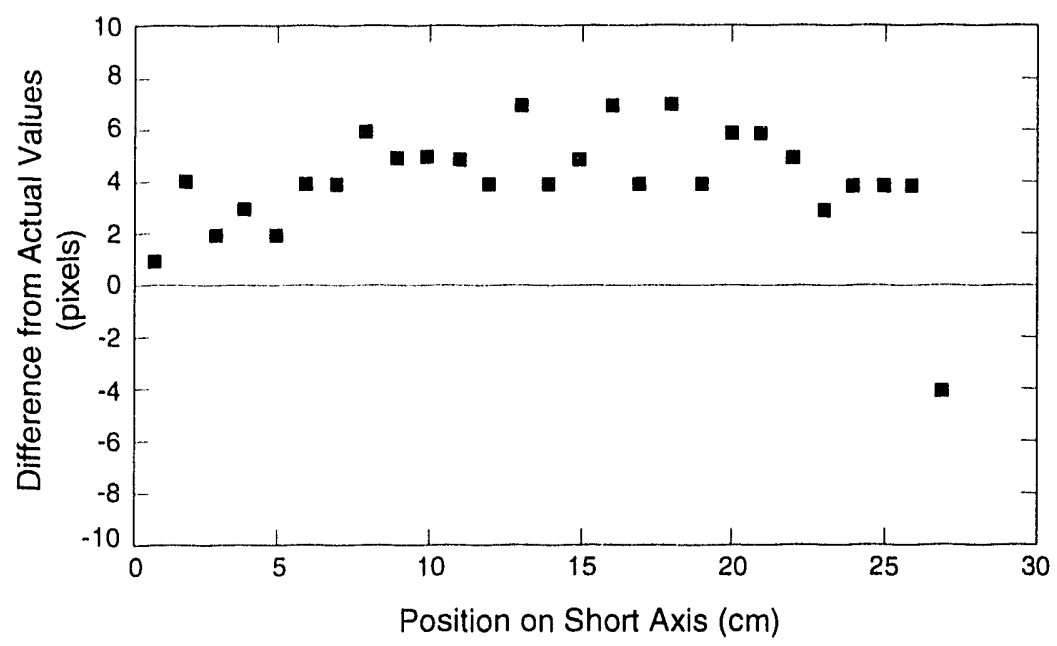

(b)

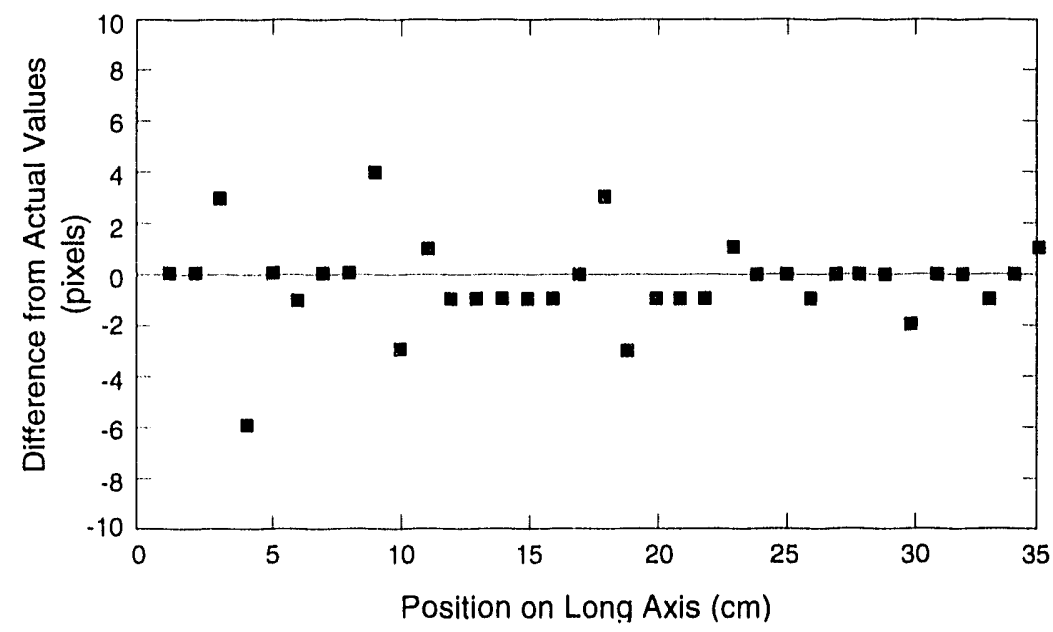

Fig. 4(a-b). Spatial distortions across the short axis (a) and the long axis (b) for the MD film scanner. 
(a)

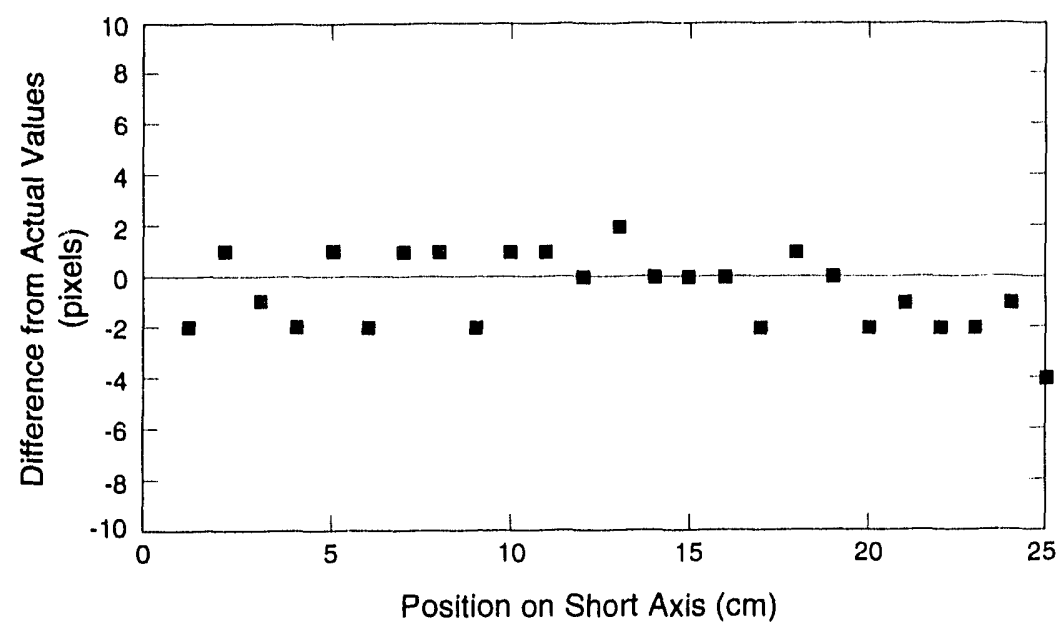

(b)

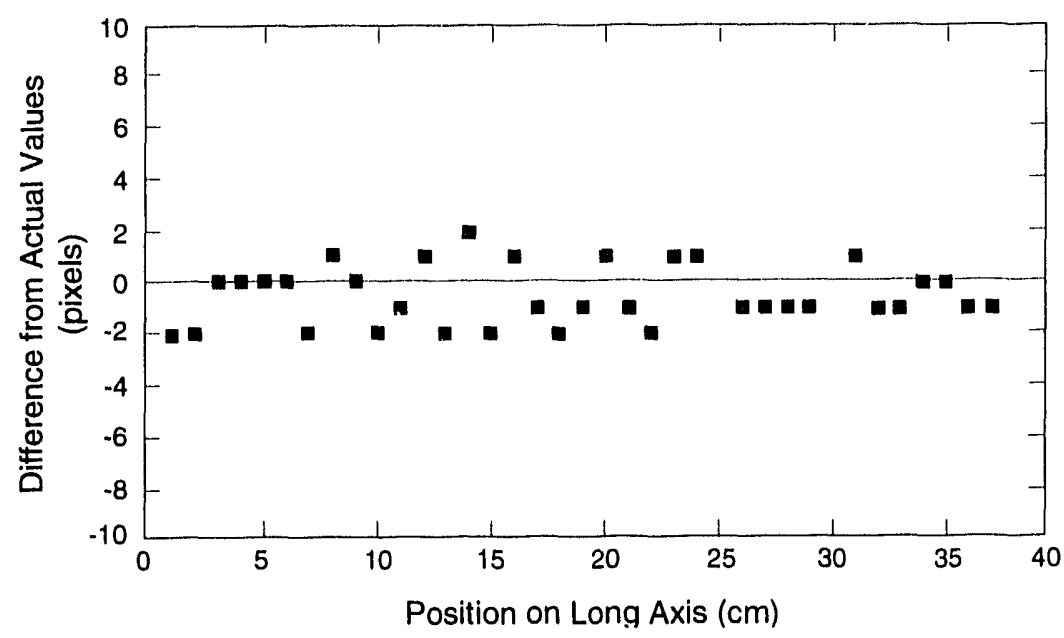

Fig. 5(a-b). Spatial distortions across the short axis (a) and long axis (b) for the MD phosphor scanner.

Within the film plane, DuPont's film scanner had low spatial distortions and averaged 140 pixels $/ \mathrm{cm}$ with a $0.966 \mathrm{std} \mathrm{dev}$ for the short axis (Fig. 6a). The average value for the long axis (Fig. 6b) was close at 139 pixels $/ \mathrm{cm}$, with a 0.772 std dev. These measurements demonstrate that this scanner can measure distances consistently and accurately.

\section{B. SNR Measurements}

Figure 7 shows the SNR values for the MD film scanner: Up to the 1.5-mrad dose, the data increases linearly, at which point the film's OD decreases the transmission of the laser light through the film so that the signal values stop increasing linearly for increasing doses. The resulting low signal values and high standard deviation of the data decrease the SNR at the higher OD. The maximum of the curve occurs at an OD of $\sim 2.0$.

Figure 8 shows a similar SNR curve for the DuPont scanner. Again, as with the MD film scanner, the curve increases steadily but then maximizes at $\sim 1.5 \mathrm{mrad}$. The maximum SNR for this scanner is about 110 . If the OD range were increased on the DuPont scanner, allowing more light transmission through the larger OD on the film, the SNR increases slightly. This feature was available only on the DuPont scanner. 
(a)

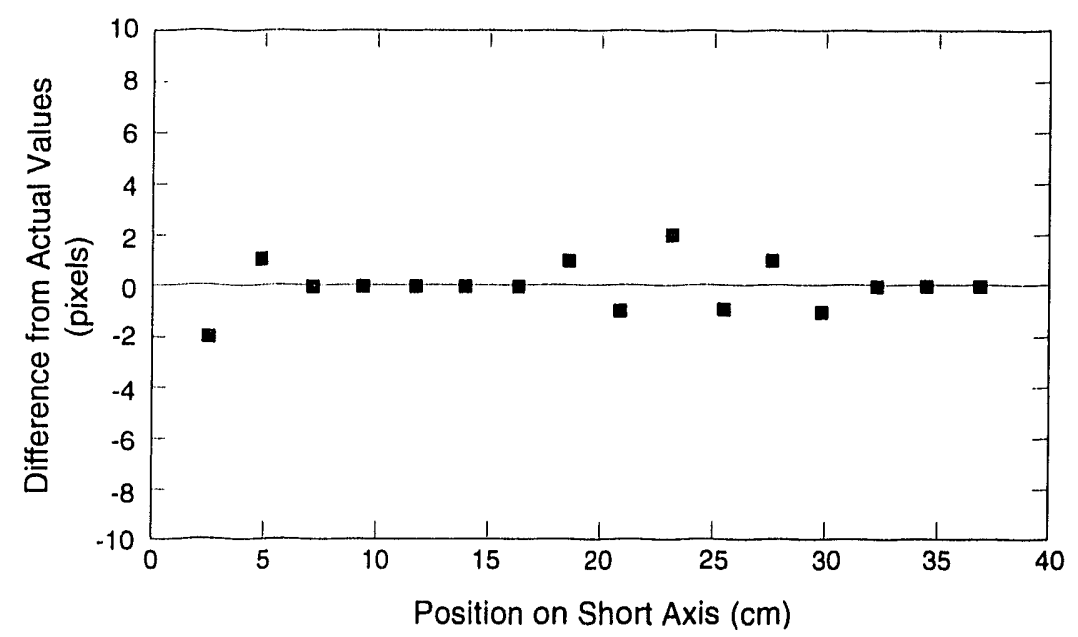

(b)

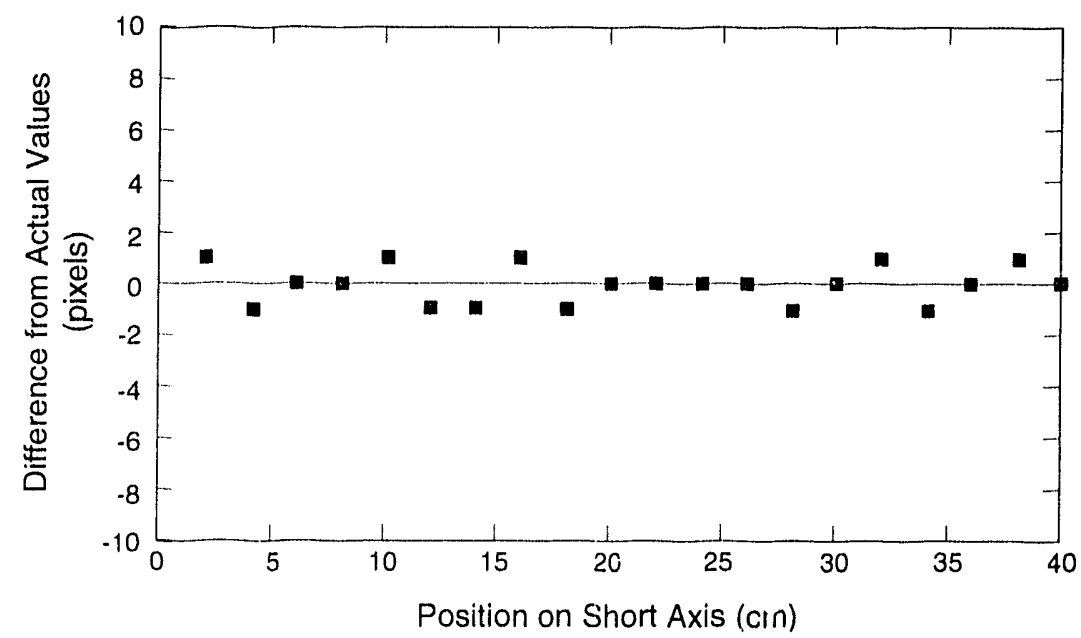

Fig. 6(a-b). Spatial distortions across the short axis (a) and the long axis (b) for the DuPont film scanner.

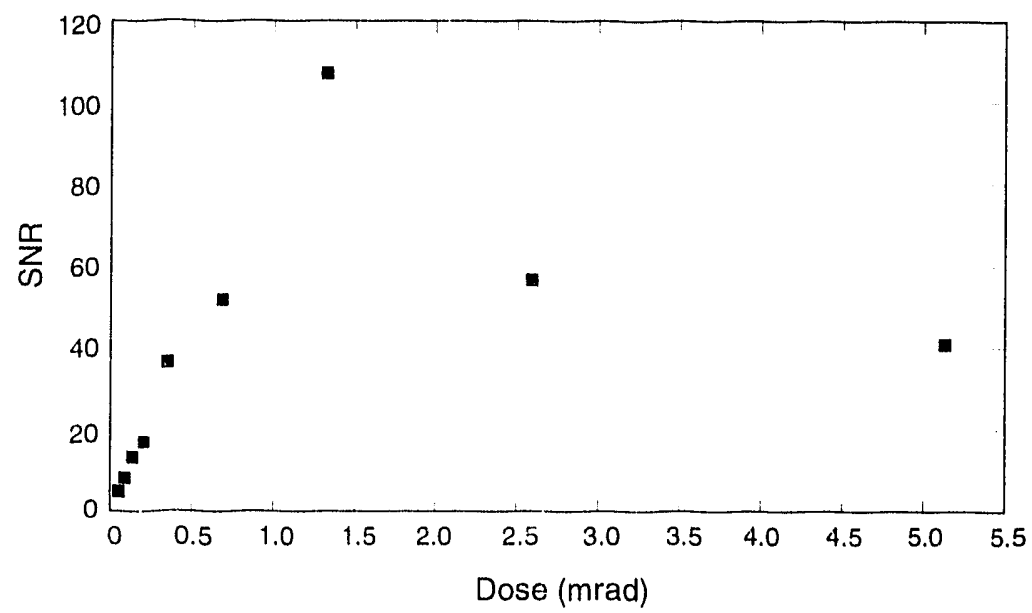

Fig. 7. SNR values for the MD film scanner. 
With its large dynamic range, the phosphor imager shows a rapid increase in SNR for lower doses then flattens out (Fig. 9). The curve continues upward for increasing doses to the phosphor plane and does not drop, as did the film scanner's SNR curve. The actual SNR values, however, are at least a factor of 2 lower than those of the film.

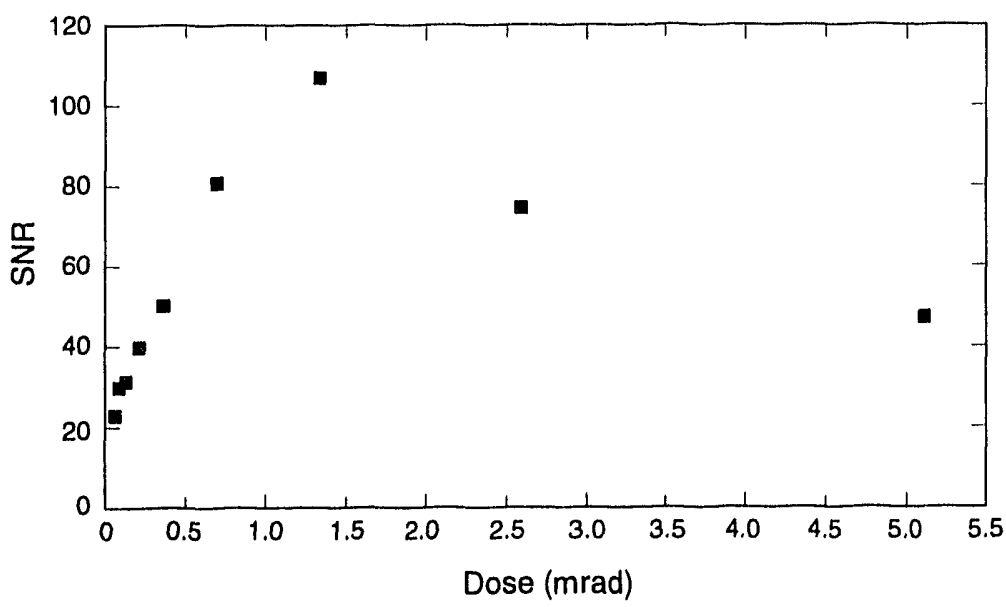

Fig. 8. SNR values for the DuPont film scanner.

Fig. 9. SNR for the MD phosphor scanner.

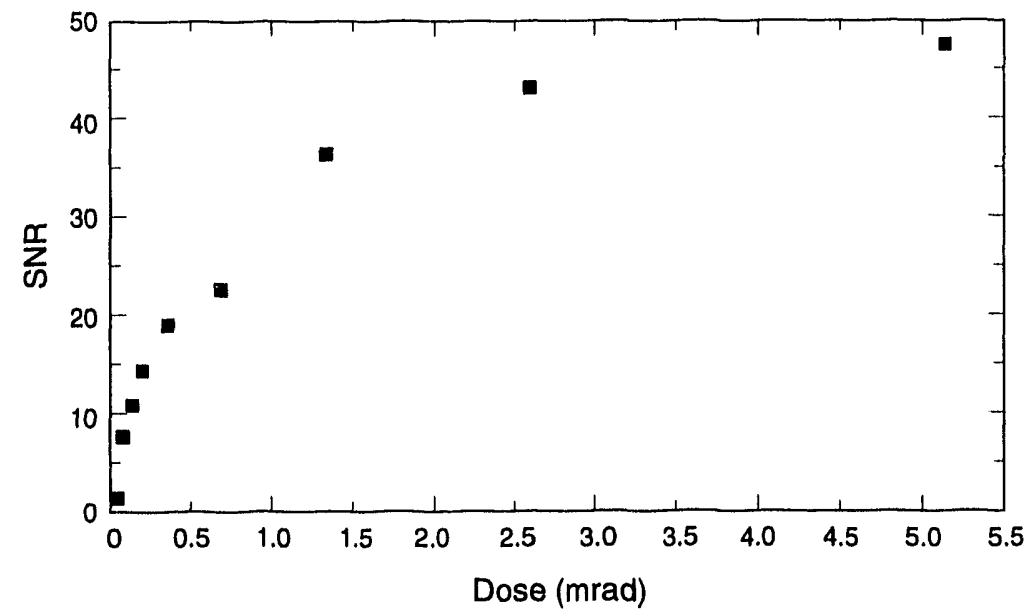

Table I compares the characteristics for all three of the scanners discussed in this report.

Table I. Scanner characteristics.

\begin{tabular}{|l|c|c|c|}
\hline Characteristic & DuPont & MD film & MD phosphor \\
\hline Scanning time $(14 \times 17 \mathrm{in})$. & $20 \mathrm{~s}$ & $\sim 2.0 \mathrm{~min}$ & $\sim 4.0 \mathrm{~min}$ \\
\hline OD range variable & yes & no & N/A \\
\hline ROI scan & yes & yes & yes \\
\hline PMT voltage variable & no & no & yes \\
\hline Data averaging real time & yes & no $^{\mathrm{a}}$ & no $^{\mathrm{a}}$ \\
\hline Pixel size variable & no & no & yes \\
\hline Spatial distortions & low & high & low \\
\hline Peak SNR & $\sim 110$ & $\sim 110$ & 50 \\
\hline
\end{tabular}

a Software has now been written that will average the pixel data after the data file is created.

b The peak SNR was found from dose range of $0.5 .12 \mathrm{mrad}$. 


\section{CONCLUSION}

All of the scanners tested are high-end performance scanners. The DuPont has good SNR ratios, scanning time, optional parameters (such as selecting OD range), and excellent spatial accuracy; however, the large size and sensitive electronics that are associated with this scanner introduce some impracticalities for field use.

The MD film scanner, on the other hand, provides a compact and rugged system that is deployable, but the spatial distortion data suggests that the scanner is not accurate for measuring distances along the film plane. Unfortunately, there are no hardware adjustments that can fix the problem, which seems to be inherent across all MD film scanners, and the necessary fixes will have to made by compensating in software.

The phosphor scanner was just recently introduced in the commercial market and has several advantages over film:

- the scanner needs no chemical processing to produce a radiographic image;

- the phosphor screen can be used more than once and is not sensitive to light except for red, which means that the phosphor can be manipulated in the light, eliminating the need for both a darkroom and developer; and

- the system has a low-dose-rate capability, which is the main advantage of the phosphor over film in field applications; thus, acceptable images can be produced using as little as a $10-\mu \mathrm{rad}$ dose at the phosphor plane (150-pkeV source).

However, there are some problems associated with the phosphor scanner:

- It produces good spatial accuracy across the film plane but the SNR data is far lower than that of film; although, the PMT voltage and laser radius can both be altered to enhance the SNR data.

- There have been some problems associated with temperature affecting the radius of the laser focal spot. To prevent loss of data, environmental controls will be needed to keep the radius constant.

The reliability of the phosphor scanner is certainly a concern. If the scanner failed in the field, there would be no other options for data collection. As a precaution, film developers, darkrooms, and film scanners will all have to be part of a field-deployable system. Film serves as a backup that can be viewed without the use of scanners. And even though the phosphor screen is excellent for low-dose radiography, the film scanner provides additional capabilities for radiography. This was demonstrated in a recent experiment in which phosphor and film (type $M$ ) were loaded into a film cassette and a ${ }^{60} \mathrm{Co}$ source was used to irradiate a complex enclosed object for $5 \mathrm{~min}$. The film captured many details, but the phosphor scanner failed to capture the innermost details, even after intense image-processing techniques were performed. This phenomenon will necessarily have to be closely investigated to determine if indeed the phosphor scanner could not distinguish the inner details. It may be possible to use some radiography techniques to enhance the response of the phosphor for high-energy sources but that has yet to be researched.

Overall, the DuPont film scanner has the best capabilites for producing high-quality images. Its portability, however, makes it unsuitable for field use. By contrast, the MD scanner lacks some of the capabilities of the DuPont scanner (for example, the ability to select an OD range or averaging data in real time) and has significant spatial distortions, which will have to be compensated for in the software, but it is very portable and has equally good SNRs. The MD phosphor 
scanner has relatively low SNRs; however, its low-dose capability, low spatial distortions and scanning characteristics (that is, being able to work in the light and no chemical processing) are important factors. In addition, software has been written that will average a specified number of pixels from the data files generated by the MD film/phosphor scanner. ${ }^{4}$ Although this averaging is not done in real time, as in the DuPont scanner, it provides a necessary tool that will decrease the data file size and increase the SNR.

Regarding performance, both the DuPont and MD scanners have been used successfully in radiography exercises. Other software development, however, can be done to enhance the user interface of the scanners. This will actually be necessary to make the scanners easier to use and to ensure that data collected from the scanners can be repeatedly used successfully.

\section{REFERENCES}

1. R. A. Morris, "Evaluation of Storage Phosphor Technology," Los Alamos National Laboratory report LA-12033-MS (April 1991).

2. Phosphor Imager Users Guide, Molecular Dynamics (1991).

3. N. Shalkowski, "Annual X-ray Protection Survey," Los Alamos National Laboratory memorandum HS-12-FTHT-93-001, to N. Nicholson (Jan. 6, 1993).

4. J. Cavender, Los Alamos National Laboratory, personal communication, August 1991. 

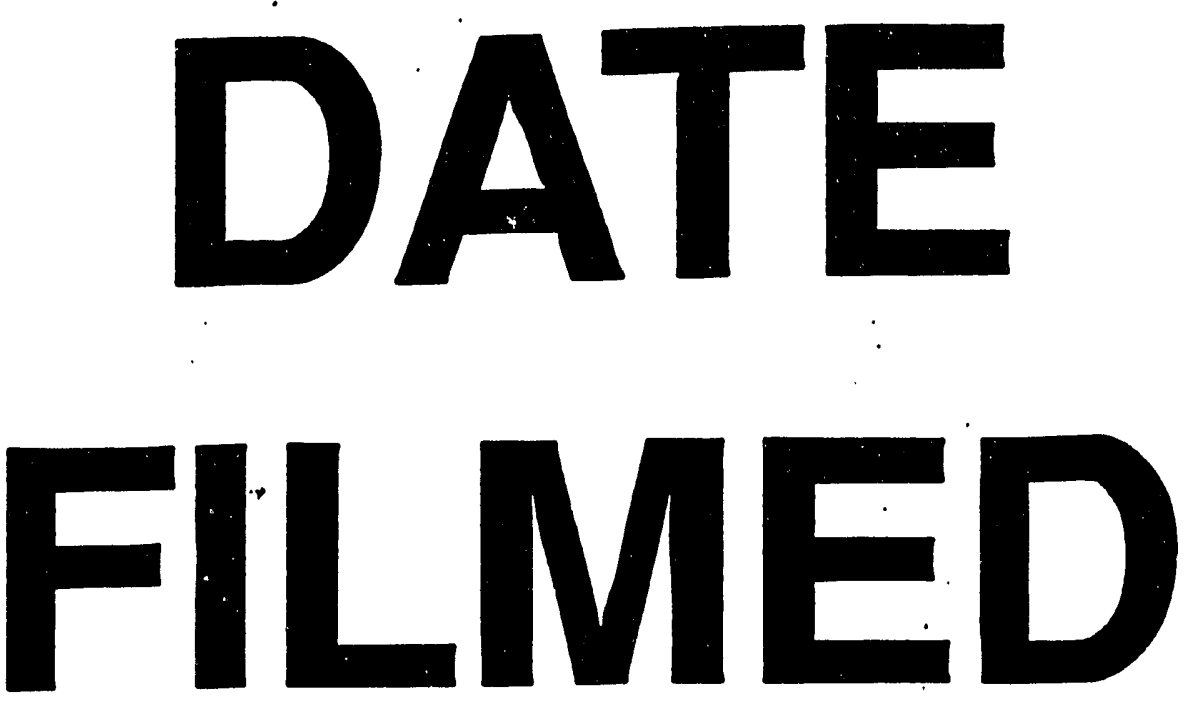

$12 / 3 / 93$
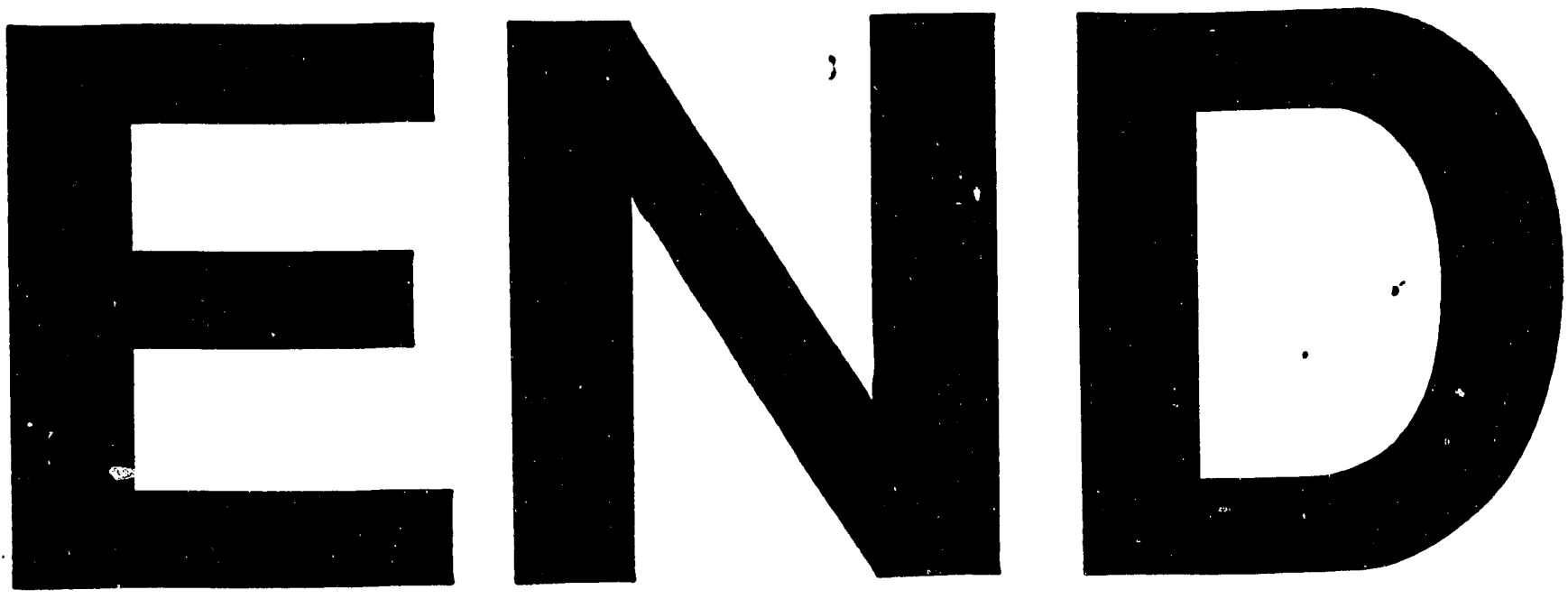
